\title{
A Comprehensive X-Ray Spectral Code for High Energy Astrophysics
}

\author{
D. A. Liedahl, K. B. Fournier, C. W. Mauche
}

\section{August 18, 2000}

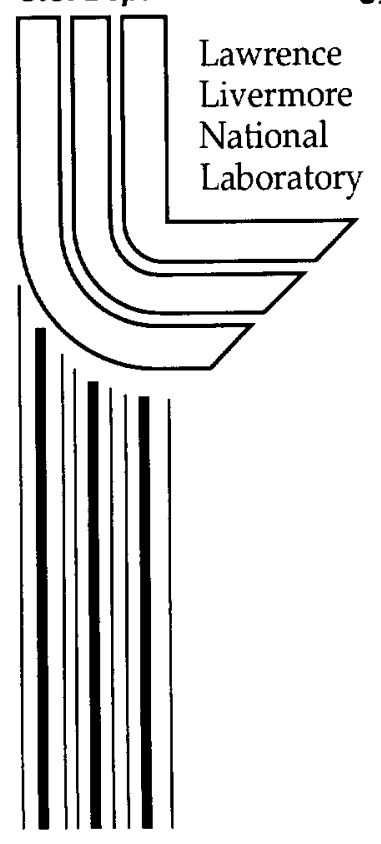




\section{DISCLAIMER}

This document was prepared as an account of work sponsored by an agency of the United States Government. Neither the United States Government nor the University of California nor any of their employees, makes any warranty, express or implied, or assumes any legal liability or responsibility for the accuracy, completeness, or usefulness of any information, apparatus, product, or process disclosed, or represents that its use would not infringe privately owned rights. Reference herein to any specific commercial product, process, or service by trade name, trademark, manufacturer, or otherwise, does not necessarily constitute or imply its endorsement, recommendation, or favoring by the United States Government or the University of California. The views and opinions of authors expressed herein do not necessarily state or reflect those of the United States Government or the University of California, and shall not be used for advertising or product endorsement purposes.

This work was performed under the auspices of the U.S. Department of Energy by the University of California, Lawrence Livermore National Laboratory under Contract No. W-7405-Eng-48.

This report has been reproduced directly from the best available copy.

Available electronically at http://www.doc.gov/bridge

Available for a processing fee to U.S. Department of Energy

And its contractors in paper from

U.S. Department of Energy

Office of Scientific and Technical Information

P.O. Box 62

Oak Ridge, TN 37831-0062

Telephone: (865) 576-8401

Facsimile: (865) 576-5728

E-mail: reports@adonis.osti.gov

Available for the sale to the public from

U.S. Department of Commerce

National Technical Information Service

5285 Port Royal Road

Springfield, VA 22161

Telephone: (800) 553-6847

Facsimile: (703) 605-6900

E-mail: orders@ntis.fedworld.gov

Online ordering: $\underline{\mathrm{http}: / / w w w . n t i s . g o v / \text { ordering.htm }}$

\section{OR}

Lawrence Livermore National Laboratory

Technical Information Department's Digital Library

http://www.llnl.gov/tid/Library.html 


\title{
A COMPREHENSIVE X-RAY SPECTRAL CODE FOR HIGH ENERGY ASTROPHYSICS
}

\author{
FINAL, REPOR'T \\ Duane A. LiedahI, Kevin B. Foumier. and Christopher W. Matuche \\ Physics Deparment, VDirision
}

ABSTRKC 7

\begin{abstract}
The aim of this project has been to develop a spectral analysis tool with a level of quality and completeness commensurate to that expected in data from the current generation of $X$-ray observatories. The code is called LXSS (Livermore X-Ray Spectral Synthesizer). X-ray-emitting astrophysical plasmas are rarely, if ever. in LTE. so we have adopted the detailed level accounting approach, in which rates for processes that populate or depopulate atomic energy levels are treated explicitly. This cotails the generation of a large quantity of atomic data. most of which is calculated using "in-house" computer codes. Calculations are henchmarked against laboratory data. and spectral models have been used to provide first-time interpretations of astrophysical $X$-ray spectra. The design of a versatile graphical user interface that allows access to and manipulation of the atomic database comprises the second major part of the project.
\end{abstract}

\section{THE NEW GENERATION OF X-RAY OBSERVATORIES}

The international commitment to advancing the field of $X$-ray astronomy is evidenced by the recent launches of NASA's Chandra X-ray Observary (Aug 1999) and the European Space Agency's X-Ray Multi-Mirror Mission (XMM: Dec 1999). Unfortunately, a third major mission, Astro-E, a cooperative effort hy Japan and the U.S.. failed to achieve orbit. The simultaneous deployment of two facility-class $X$-ray satellites. comprising several billion dollars of space hardware, heralds a potential revolution in the field.

The mission objectives of Chandra. and $X M M$ are predominantly spectroscopic: they carry spectrometers that. combined. cover the 1-160 $\mathrm{A}$ hand, with large collecting areas (hundreds of squate centimeters) and high spectral resolving powers $(\lambda / \Delta \lambda$ of several hundred). These missions thus offer astronomers and astrophysicists their first opportunity to obtain high-quality $X$-ray spectra from extrasolar $X$-ray sources. With their projected ten-year lifetimes. thousands of $X$-ray datasets will he generated. representing almost every class of celestial ohject: stellar coronate. supernova remnants. normal galaxies. clusters of galaxies. white dwarfs neutron stars. and hlack holes. An understamding of spectral formation leads to an understanding of the source phistes.

\section{ASTROPHYSICAL X-RAY LINE SPECTROSCOPY AND SPECTRAL SYNTHESIS}

In terms of scientific data. the most salient aspect of Chandra and $X M M$ is that. for the lirst time. $\mathrm{X}$-ray lime spectroscopy will hecome the central theme of $X$-ray astronomy. It has heen remarked that we are ahout to see the field mathe a transition from astronomy to astrophysics. Fateilitang this transition. humerer. is problematic, a lesson 
brought to us by the previous major X-ray mission. the Adranced Satellite for Cosmology and Astroplessics (ASCA: launched in 1993). which was a joint effort of Japan and the U.S. Even though the CCD spectrometers aboard ASCA lacked the high resolving powers of Chamdra and $X M M$, it became evident that the theoretical tools available for spectroscopic analysis were not up to the task.

Theoretical models of X-ray line spectra are generated by computer codes called spectral syuthesis codes (SSCS). which provide the means to translate observed spectra into statements of the source physics. In essence. an SSC allows access to and manipulation of one or more dalabases contaning atomic quantities. The atomic yuantities consist of energy level structure. radiative decay rales. collisional excitation rates. photoionization cross-sections. and so forth. This information is used to calculate level population distributions for each ion at a given temperalure and density. from which follows the emission-line spectrum. Also calculated are the continum radiation processes: ion-electron bremsstrahlung. two-photon decay. and radiative recombination. Detailed data for the elements hydrogen, helium, carbon, nitrogen, oxygen. neon. magnesium. silicon. sulfur. argon, calcium. and iron are mandatory in order to generate a reasonable model spectrum of a typical celestial X-ray source. Of somewhat less importance, but required to achieve completeness. are data for the elements sodium, aluminum. and nickel. For each element. every charge state with hound electrons falls within the purview of a complete SSC. giving a total of 179 ions.

Modern SSCs are not "hardwired" to use a particular set of atomic data. Rather. they access databases that are modular in design; as improved atomic data become avalable, the obsolete data are simply replaced. requiring no explicit modifications to the SSC. Data come to SSC developers in a variety of formats. and come from a mix of experimental and theoretical work. Considerable effort is expended in maintaining familiarity with the literature. acquiring new atomic data, and reformatting it into a form that the SSC can ingest. Thus a division of labor has evolved: data generators (masthe a hundred scientists) provide data to SSC developers (a few scientists). who facilitate access to atomic data for users (thousands of scientists). This division is not stricly adhered to. of course. but it adequately represents the "culture" of X-ray astronomy.

\section{THE LXSS PROJECT}

In the early 90 s, coming into the field primarily as observers and modelers, we hegan also to play the role of generators of atomic data to seteral of the major SSC development groups. Slow turnaround times and cuality control issues surfaced as problems. however. Moreover, although we were in the habit of regularly generating state-ot-the-art atomic models. they were usually designed for a specific problem. and lacked generality. We were left with a large non-uniform. unwieldy database. unsuitable for astrophysical datal analysis. These various factors led us to the conclusion that our interests could he better served by constructing our oun SSC. Our team has significant werlap with all three groups delineated above: as ohservers/analysts. we are familiar with the desired scope and functions of SSCs: a data generators. we can assemble a highly-customized datahase and impose our own standards of quality: as SSC developers. we are able to design a code that facilitates our own research activities. 


\subsection{Construction}

With LDRD funding we hegan work on the Livermore X-ray Spectral Synthesizer (LXSS). The lasks laid out for us were:

(1) generation of the bulk of the atomic datahase using LLNL computer codes

(2) compilation. critical assessment. and incorporation of atomic data that cannot be generated at

LI.NL in a timely manner

(3) design of a user interface that will allow rapid manipulation of the database

\subsubsection{Collisionally Ionized Plasmas}

A large part of the database has been generated using the HULLAC (Hebrew University/Lawrence Livermore Atomic Code) suite. HULLAC gives us the means by which to quickly generate atomic structure calculations. radiative and autoionization transition rates, and electron-ion collisional excitation rate coefficients. Along with the charge state distributions of each element at a given temperature. these constitute the essentiat components of an SSC designed for application to plasmas in coronal ionization equilibrium (CIE). In fact, most of the "production" codes are designed for this environment. Coronal ionization equilibrium applies to stellar coronae, clusters of galaxies. and probably interstellar media. The shocked gas in supernova remnants may be in a transient phase of ionization disequilibrium. hut the level population kinetics is much the same as in CIE. LXSS includes a differential equation solver that determines the charge state distribution as a function of time under transient conditions.
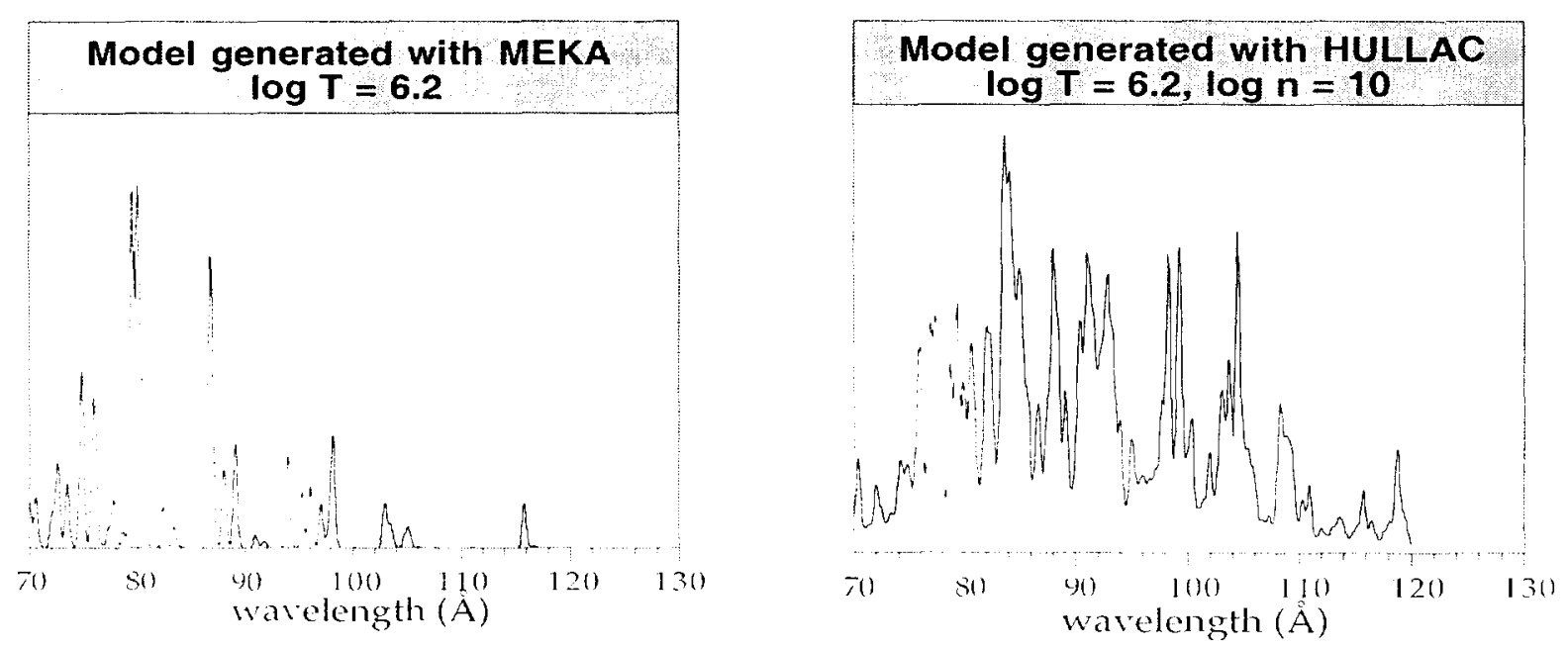

Figure 1. Comparison of model spectra predicted by two models: (left pameth the MEKA code. which is currently the best puhlicly anabic SSC in this spectal range and (righ pemet) LXSS. This comparison cxemplities the "missing line" problem in

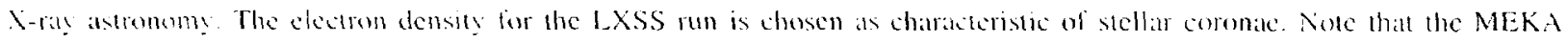
motel makes mo provision for elensity dependence. since it deses not invert at rate matris for the lesel populations. 


\subsubsection{Photoimised Plasmas}

The other important class of ionization dynamics is photoionization equilibrium. in which photons. rather than electrons. are primarily responsible for ionizing atoms. In photoionized gas. the charge state fraction of a given ion peaks at a temperature that is much lower than in CIE. Radiative and dielectronic recombination dominate the level population kinetics in a photoionized gas. In addition to those listed in \$3.1.1. the essential atomic physics ingredients for these calculations are photoionization cross-sections, which permit the calculation of radiative recombination cross-sections from a detaled halance relationship. We have constructed atomic and spectral models appropriate for this regime, and included them in LXSS. which makes LXSS unique with respect to other SSCS. While it is true that the class of codes called photoionization codes include recombination spectral models. the LXSS models are the current state-of-the-art. Photoionization equilibrium applies to quasars. Sevfert galaxies. and $\mathrm{X}$-ray binaries.


Figure 2. Comparison of LXSS iron spectra for two types of ionization equilibria: (left panel) collisional ionization equilibrium and (righ paneh) photoionization equilibrium. The charge state distribution in either case corresponds to conditions for which $\mathrm{C}$ like Fe XXI altains its peak. The model spectrum is folded through a gaussian resolution kernel chosen to approximate the spectral resolving power of the $X M M$ gratings.

\subsubsection{Aromic Database}

Table 1 lists some of the ingredients of LXSS. and gives an indication of the large degree to which the LXSS datahase is generated with LLNL resources. Alomic data are stored in a hinary format known as FITS (Flexible Image Transport System) formalt. for easy interface with filling packages provided by NASA centers. We have written several "pipeline processing" coles in IDI. (Interactive Data Language) to facilitate uniform data conversion and storage. An extensive IDL graphical user interface. which allows access to a number of simulation and display functions. and a database query function. are now operallonal. 
The ability to generate the vast majority of the required atomic data ourselves gives us several advantages over other SSC groups. For example. we choose the atomic model size and the physical parameter space over which it validly operates. rather than heing forced to settle for what is avalable in the literature. Since we can create and format atomic data much more rapidly than the community of generators. lag times are much shorter.

TABLE 1 Alomic physics and modeling options contained in LXSS classified according to origin.

\begin{tabular}{|c|c|c|}
\hline Feature & $\begin{array}{l}\text { Internal } \\
\text { Sources }\end{array}$ & $\begin{array}{l}\text { External } \\
\text { Sources }\end{array}$ \\
\hline atomic atructure & $\checkmark$ & \\
\hline radiative transition rates & $\checkmark$ & \\
\hline two-photon transition rates & & $\checkmark$ \\
\hline autoionization rates & $\checkmark$ & \\
\hline photoionization cross-sections & $\checkmark$ & \\
\hline electron-ion impact cross-sections & $\checkmark$ & \\
\hline proton-ion impact cross-section & & $\checkmark$ \\
\hline collisional ionization rates & & $\checkmark$ \\
\hline dielectronic recombination rates/satellite spectra & $\checkmark$ & \\
\hline bremsstrahlung continuum & & $\checkmark$ \\
\hline radiative recombination continuum & $\checkmark$ & \\
\hline charge state distribution & $\checkmark$ & $\checkmark$ \\
\hline$\ddot{j}$-averaging and $n$-averaging & $\checkmark$ & \\
\hline line and ion continuum opacity & $\checkmark$ & \\
\hline graphical user interface & $\checkmark$ & \\
\hline
\end{tabular}

\subsection{Benchmarking}

Even the most sophisticated computer codes run up against limitations. In calculating attomic energy level structure. for example. an intrinsic error $\Delta E$ translates into a wavelength error $\Delta i$ that scales as $i^{2}$. In other words, wavelength predictions get rapidly worse moving from the X-ray hand towards the ultraviolet hand. In the $\sim 10 \AA$ region. HUL L.XC wavelenghs have heen shown to he accurate typically to within roughly a part in 1000 , while near $100 \AA$

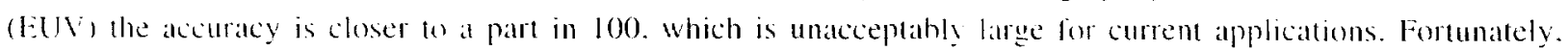
errors of this urder are not callistophic: code output can he adjusted "by hand" to conform to experimental data. 
where that data exists. and where it is reliable. We have benelitted from close collaborations with experimentalists involved with tokamaks and the Electron Beam Ion Trap (EBIT) at LLNL. as well as ohservers involved with orbiting observatories such as the Exweme Ultraviolet Explorer (EUVE) and ASCA. Data accuired with these facilities provide suitahle testheds and standards against which to evaluate and improve the quality of our calculations.

Without going into great detail. We can say the LXSS models have fared extremely well in comparisons against laboratory data. We have found cases where the omission resonant excitation in our models leads to errors in predicted intensities of order $30 \%$. most noticably in the EUV spectral range. Shortcomings of this type are corrected most easily by supplementing HULLAC data with the results of more exact calculations (see Table 1) which use. for example. an R-matrix code. It is worth emphasizing that in the harder X-ray hand $(\lambda<35 \AA)$. EBIT experiments show that HULLAC (hence. LXSS) models perform at a high level of accuracy. In collaborations with groups in England. Holland. and the U.S., we are applying small wavelength corrections to our models in order to conform to the highly precise measurements obtained in tokamaks. EBIT. the solar corona. and stellar coronale which are now available.

\subsection{Applications}

Although LXSS has yet to be released. we are already using models that constitute its raw ingredients to analyze and interpret spectra from both celestial and terrestrial sources. An appreciation of the scope of these activities can be gained through an examination of the titles listed in Part 5. Two highlights: (1) Using high-resolution X-ray spectroscopy of neon and argon emission from a well-diagnosed magnetically confined fusion plasma. it was demonstrated that resonance enhancement of direct collision rates needs to be taken into account in simulating transitions between excited atomic states. This is crucial for correctly implementing spectroscopic diagnostics of local particle transport in tokamaks. which. in turn. is required in order to understand the overall energy halance inside the plasma. (2) In X-ray astronomy, we have obtained, for the first time. a hroad hand spectral fit to the discrete X-ray spectrum of a neutron star X-ray binary using a spectral model hased on actual atomic physics. rather than an arbitrarily adjustable empirical model. This has allowed us to derive physical constraints of the accretion flow in the high-mass X-ray pulsar Vela X-1. Since the LXSS project was begun prior to the launches of Chandra and $X M M$. the primary scientific drivers for this project. the most exciting applications are yet to come.

\section{OUTLOOK}

The construction phase of IXSS as an astrophysics interpretive tool is near an end. While it $\mathrm{s}$ true that such codes can never really he finished. since new developments in atomic physics continually lead to improvements in the quality of the atomic database. we have reached our first major plateau. Our close involsements in X-ray alstronomy and laboratory spectroscopy will provide a wealth of applications. We have heen awarded a number of Pl Guest Observerships on Chandra. XMM. and Astro-E. and are participants in the XMM Guaranteed Time program (we were extremely successfut in the Cycle I review of the ill-fated Astro-E.). Over the next few years. we thus foresee many excellent opportunities to exercise the full capability of LXSS. We expect LXSS to evole to the point where. in addition to astrophysical applications. it supports all internal $X$-ray spectroscopic modeling and interpretation. 


\section{SUPPORTED PUBLICATIONS}

Bautista. M.. Kallman. T. Angelini. L. Liedahl. D. 1998. "Hydrogenic Ion Emission in the Spectra of Low-Mass XRay Binaries." The Astrophysical Journal (UCRL-JC-1.37278).

Brown. G.. Beiersdorfer. P.. Liedah1, D.. Kahn. S. and Widmann. K. 1998. "Laboratory Measurements and Modeling of the Fe XVII X-Ray Spectrum." The Astrophrsical Journal. 502. 1015 (UCRL-JC-128877).

Beiersdorfer. P.. Utter, S.B., Brown, G.V., Liedahl. D.A.. Mauche. C.W.. Kahn. S.M.. Dupree, A.K.. Brickhouse. N.S.. Mewe. R.. and Kaastra. J.S. 1998. "Spectral Catalogue of the Intermediate Ionization States of Iron in the Extreme Ultraviolet." Proc. NASA Workshop on Laboratory Space Science (UCRL-JC-130832).

Brickhouse. N.S.. Dupree, A.K., Edgar, R.J., Liedahl, D.A.. Drake. S.A.. White. N.E., and Singh, K.P. 2000. "Coronal Structure and Abundances of Capella from Simultaneous EUVE and ASCA Spectroscopy." The Astrophrsical Jourmal, 530, 387 (UCRL-JC-139171).

Foumier. K.B.. Finkenthal. M., Pacella, D., May, M.J.. Soukhanovskii. V.. Mattioli. M.. Leigheb, M., and Rice, J.E. 2000. "Tokamak Spectroscopy for X-Ray Astronomy," Proceedings of the Workshop on Atomic Data Needs in X-ray Astronomy, ed. M. Bautista and T. Kallman (UCRL-JC-137837).

Fournier. K.. M. Cohen. W. Goldstein, "Dielectronic Recombination Rates in H-like Ar ${ }^{17+}$ to Ne-like Ar ${ }^{8+}$," Physical Revien A, 56. 4715 (UCRL-JC-127789).

Fournier. K. M. May. D. Pacella. B. Gregory, M. Finkenthal. W. Finkenthal, "Calculated Radiative Power Losses from Mid- and High-Z Impurities in Tokamak Plasmas," AIP Conference Proc.. 4+3. 73 (UCRL-JC-130183).

Fournier. K.. M. Cohen, M. May. W. Goldstein, "Radiative Cooling Rate and Charge State Distribution for Argon in a Low Density Plasma." Atomic Data and Nuclear Data Tables (UCRL-JC-129529).

Gu. M.F. Beiersdorter. P.. Brown. G.V.. Kahn. S.M. Liedahl. D.A.. Reed. K.J.. and Savin. D.W.. 1999. "Lahoratory Measurements of Resonant Contributions to Fe XXIT Line Emiscion." Phisica Saripta. TS0. 310 (l) CRI-JC-132214).

Kahn. S.M. Gu. M.F. Savin. D.W.. Beiersdorter, P.. Brown. G.V.. Decaus. V.. Liedahl. D.A.. Utter. S.B.. and Widmann. K. 1908. "Laboratory X-Ray Spectroscopy Experiments in Support of NASA's X-Raly Satellite Missions." Proc. NASA Workshop on Laboratory Spare Scitence (L CRL-JC-130129). 
Liedahl. D.A.. Beiersdorfer. P.. Brown. G.V.. Utter. S.B.. Kahn. S.M. Gu, M.F. and Savin. D.W. 1998. "A Synergistic Approach to Modeling X-Ray Spectra," Proc. of the NASA Workshop on Laboratory Space Science (UCRL-JC-131403).

Liedahl. D.A.. Sako, M. Wojdowski. P.S., Paerels. F.. and Kahn. S.M. 2000. "X-Raly Line Spectra in Marsive XRay Binaries." Revista Mexicana de Astronomia y Astrofisica. Serie de Conferencias, in press (UCRL-JC$138+65)$.

Liedahl. D.A. 2000, "The Completeness Criterion in Atomic Modeling." Proceedings of the Workshop on Atomic Datu Needs in X-ray. Astronomy. ed.. M. Bautista and T. Kallman (UCRL-JC-139120)

Liedahl. D.A. 1999. "The X-Ray Properties of Photoionized Plasmas and Transient Plasmas." in X-Ray Spectroscopy in Astrophysics. eds J. van Paradijs and J. Bleeker. Springer Lecture Notes in Physics. p. 189 (UCRL_JC-132453).

Liedahl. D. A. 1999. "Spectroscopy With the New Generation of X-ray Observatories." Phisica Scripta. Vol. T83, 110 (UCRL-JC-132424).

Mattioli. M., "Experimental and Simulated Neon Spectra in the 10-nm Wavelength Region from Tokamak and Reversed Field Pinch Plasmas." Physical Review E, 60, 4760 (UCRL-JC-134074).

May, M., et al., "Benchmarking Atomic Physics Models for MCF Plasma Physics Experiments." Rev'. Sci. Instrum. (UCRL-JC-132593).

May, M.J.. Fournier. K.B.. Pacella. D., Kroegler, H., Rice, J.E., Gregory, B. Finkenthal. M.. Moos. H.W.. Mazzitelli. G.. and Goldstein. W.H. 2000. "Ohservations of the Ultraviolet and X-Ray Brightness Profiles and Cooling Rates of $\mathrm{Kr}$ and $\mathrm{Ar}$ in Magnetically. Confined Fusion Plasmas." Physical Revierl E, 61. 3042 (LCRLJC- 135407$)$.

Pacella. D. ct al., "Measurement of the Radiative Cooling Rate for Krypton and Argon and Their Profiles in the

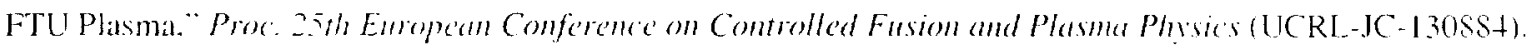

Rice. J., at al. "The Rydtherg Series of Helium-like Cl. Ar. and S and their lligh-n Sittellites in Tokamak Plasmas." New Journat of Phisis. 1. 19.1-19.27 (UCRL-JC-135505).

Sako. M.. Kahn. S.M.. Paderels. F.. and Liedahl. D.A. 2000. "The Physical Conditions in the X-ray Emission Line Region of the Circinus Galat!." The Astrophesical Joumal, submitted (UC RI-JC-139125). 
Sako. M.. Liedahl, D.A.. Paerels. F.. and Kahn. S.M. 2000. "The X-Raly Spectrum and Glohal Structure of the Stellar Wind in Vela X-1," The Astrophesical Jonmal. 525.921 (UCRL-JC-137276).

Savin. D.W.. Beck, B.. Beiersdorter. P.. Kahn. S.M.. Brown. G.V.. Gu. M.-F.. Liedahl. D.A.. and Scofield, J.H. 1999. "Simulations of a Maxwellian Plasma Using an Electron Beam Ion Trap." Phisica Saripta. T80. 312 (UCRL-JC-131735).

Wargelin. B.. Beiersdorfer. P.. Liedahl, D.. Kahn. S., and von Goeler. S. 1998. "Ohservation and Modeling of Highn Iron L-Shell Lines From Intermediate Ion Stages," The Astrophtsical Joumat. 496, 1031 (UCRL-JC-128010). 